\title{
COVID-19 vaccines: Equitable access, vaccine hesitancy and the dilemma of emergency use approvals
}

\begin{abstract}
Coronavirus disease 2019 (COVID-19) is a viral disease caused by severe acute respiratory syndrome coronavirus (SARS-CoV-2). Although behavioural preventive measures such as the wearing of masks and physical distancing are effective in curbing the spread of the virus, the second surges being experienced globally make it clear that the development and uptake of COVID-19 vaccines is necessary for long-term control of the pandemic. Moreover, some countries may be on the brink of a third surge. ${ }^{[1]}$ To date, while not as yet peer-reviewed, four vaccines have been announced to be $90 \%$ or more effective. ${ }^{[2]}$ The DirectorGeneral of the World Health Organization (WHO), Tedros Adhanom Ghebreyesus, has stated recently that the scientific community has set a new standard for vaccine development, in that no vaccine in history had been developed as fast as these. ${ }^{[3]}$ In this race for a successful vaccine, several ethical issues emerge.
\end{abstract}

\section{Equitable access}

Inequalities in vaccine access could mean that many poor regions in the world will be last in the queue for effective vaccines. A study published early in November this year revealed how rich countries were well into a 'shopping spree' for COVID-19 vaccines. A global assessment of purchasing agreements for the vaccine showed that high-income countries and a few middle-income countries with manufacturing capacity had already purchased almost 3.8 billion doses, with options for another 5 billion. The USA already had agreements to buy up enough doses for $230 \%$ of its population, and with time could control about 1.8 billion doses, i.e. a quarter of the world's supply. This COVID-19 vaccine shopping and hoarding means that the populations in many of these countries could be vaccinated several times over while billions of people in poorer countries would have to wait until the middle of the decade, as it will possibly take about 3 - 4 years to manufacture adequate amounts to cover the world's population. ${ }^{[4]}$ This is reminiscent of the H1N1 influenza pandemic in 2009 , when rich countries bought nearly all the vaccines that were available. It was only after these countries received adequate supplies that low- and middle-income countries managed to get access, ${ }^{[5]}$ with the vaccines arriving in most of these countries when the pandemic was over. Concerns of a repeat of this vaccine nationalism led to the WHO spearheading a coalition, the COVID-19 Vaccine Global Access (COVAX) facility with the Global Alliance for Vaccines and Immunisation (Gavi), and the Coalition for Epidemic Preparedness Innovations (CEPI), in an ambitious effort to create equitable access to effective vaccines globally. The COVAX facility is a global risk-sharing mechanism for pooled procurement and equitable distribution of the vaccines when registered for use. ${ }^{[6]}$ However, concerns regarding this initiative include the fact that several signatories to the facility, including Canada, the UK and the European Union, by negotiating bilateral deals directly with industry, undermine COVAX's goal to counter vaccine nationalism, ${ }^{[4]}$ and hence pay lip-service to its principles of global equitable access and fairness. In addition, volumes of vaccines available to countries, and financing and pricing mechanisms, remain unclear. Furthermore, independent civil society groups have highlighted the reluctance of the facility to engage with them, and it was only after months of lobbying and infighting that COVAX agreed to include them in their crucial structures. ${ }^{[7]}$

In Africa, following a meeting convened by the African Union and the Africa Centres for Disease Control and Prevention (Africa CDC) with about 3000 high-level stakeholders in June this year, the COVID19 Development and Access Strategy was released by the Africa CDC in August. The focus of the strategy is prevention of transmission of the virus, of deaths and of social and economic harm. Its key objectives are to accelerate African involvement in the clinical development of vaccines, to ensure African countries can access a sufficient share of the global supply and to remove barriers to widespread delivery and uptake of effective vaccines across the continent. ${ }^{[8]}$ While there are frameworks for equitable access of the vaccines globally and in some regions of the world, Africa is yet to develop its framework that includes not only African indigenous values and voices, but also African realities. It is critical that vaccine distribution and storage challenges are tackled urgently on the continent.

\section{Vaccine hesitancy and vaccine confidence}

Once vaccines are available for use, achieving herd immunity is largely dependent on their willing uptake by populations globally. Initiatives to allocate the vaccines fairly will not be successful if people are not willing to receive them. A recent survey conducted on behalf of the World Economic Forum revealed that only $64 \%$ of South Africans surveyed would accept a COVID-19 vaccine if it became available. This is lower than the $74 \%$ global average of adults surveyed in 27 countries who said they would accept a vaccine. Only $29 \%$ of the $64 \%$ agreed strongly to a vaccine, while $71 \%$ 'somewhat' agreed. Fear of adverse events, concerns about safety and a lack of confidence in the effectiveness of the vaccine were the two most cited reasons. Distrust in the vaccines mirrored lack of trust in public health institutions, with $32 \%$ of South Africans saying they had no trust in hospitals and clinics. The overall figure for Africa was $27 \%{ }^{[9]}$ This one-third deficit in accepting the vaccine could severely compromise the effectiveness of any COVID-19 vaccine rollout programme. Reasons for vaccine hesitancy include: the novelty of the disease; the unusually rapid speed of vaccine development; mistrust of science and health experts by some; emotional detachment due to fatigue as a result of the 'infodemic' and the uncontrollable nature and prolonged uncertainty of the pandemic; politicisation of the vaccine and its safety and efficacy standards by some groups; and conspiracy theories by antivaxxers. In addressing vaccine hesitancy and fostering vaccine confidence, it is critical that evidence-based communication strategies are used. ${ }^{[10]}$ It is alarming that vaccine disinformation is on the increase. It is essential that trust is built up to offset high levels of public health misinformation and anti-vaccination sentiment. Critical to this is that communities are able to access accurate information that is backed by evidence in order to make informed decisions on the vaccines.

\section{The emergency use dilemma}

Following the recent positive results from the of COVID-19 vaccines clinical trials, 'emergency use' approvals are being considered. This 
would mean tens of millions of people could be immunised. To this end, Pfizer and BioNTech have already commenced working towards regulatory permission. Regulators have the added responsibility of scrutinising the results so as to make decisions as to which groups should be allowed to be vaccinated, based on safety criteria. To date, none of the four vaccines announced to be effective have been peer-reviewed, and concerns regarding vaccine reactogenicity and durability abound. Scientists have raised questions as to whether ongoing clinical trials will be compromised in this way, because as soon as the vaccine is granted emergency approval, there will be a need to offer the immunisation to trial participants who were in the placebo arms. This could result in data being inadequate to establish long-term outcomes such as durability, safety and whether there is also protection against the disease, and not just infection. Some have proposed that because of the dire need, the vaccine development dilemma could be addressed by following up participants who initially received the placebo and then the vaccine, as a separate group, with the vaccine's long-term efficacy and safety monitored and compared between the groups. While the issue of bias is acknowledged, this is not a new approach, and has been done previously to determine the length of protection offered by the first shingles vaccine. ${ }^{[11]}$

\section{Conclusion}

The race for the COVID-19 vaccine is on - for both development and purchasing by rich countries at the expense of global equitable access. Disinformation poses a serious threat to achieving herd immunity. Regulatory and scientific dilemmas abound. For us in Africa, a concerted effort from all countries in the region is required to develop a co-ordinated strategy to access the vaccines, and also to finance, develop, manufacture and deliver the vaccines throughout the continent. ${ }^{[12]}$ Evidence-based communication strategies drawing from African voices are critical to address vaccine hesitancy and foster vaccine confidence. It remains to be seen how African regulators will respond to emergency use requests. In addressing the ethical issues, early engagement with relevant stakeholders, among which community and civil society organisations are critical, is pivotal. Transparency, integrity and trust are key.

\section{Ames Dhai}

Editor

ames.dhai@wits.ac.za

S Afr J Bioethics Law 2020;13(2):77-78. https://doi.org/10.7196/ SAJBL.2020. v13i2.754

1. Kyodo Japan's corona virus cluster-busting approach nears its limit. Japan Times, 2020. https://www.japantimes.co.jp/news/2020/11/23/national/sciencehealth/japan-coronavirus-clusters $/$ ?utm_source $=$ Nature + Briefing \&utm campaign=bdd2fb01a0-briefing-dy-20201126\&utm_medium=email\&utm term=0_c9dfd39373-bdd2fb01a0-44142809 (accessed 29 November 2020).

2. Cohen J, Travis J. Another COVID-19 vaccine success? Candidate may prevent corona virus transmission too. Science, 2020. https://www.sciencemag.org/ news/2020/11/another-covid-19-vaccine-success-candidate-may-preventfurther-coronavirus-transmission (accessed 29 November 2020).

3. VOA News. WHO Chief calls for equal access to COVID-19 vaccines. VOA, 2020 https://www.voanews.com/covid-19-pandemic/who-chief-calls-equal-accesscovid-19-vaccines (accessed 29 November 2020).

4. Launch and Scale Speedometer. Africa: New study shows rich country shopping spree for COVID-19 vaccines could mean fewer vaccinations for billions in low income countries. All Africa, 2020. https://allafrica.com/stories/202011020103. html (accessed 29 November 2020).

5. Usdin S. COVAX created to try to avoid global bidding frenzy for COVID-19 vaccines. Biocentury 2020;1-3.

6. Global Alliance for Vaccines and Immunisation. COVAX. Gavi, 2020. https://www. gavi.org/covax-facility (accessed 29 November 2020).

7. Johnson T. Accountability and agitation for equitable access: Keeping it real in the race for a COVID-19 vaccine. UNESCO National Bioethics Committee Webinar: COVID-19 Vaccines: Equity and access. https://www.facebook.com/HealthZA videos/288088219257823/ (accessed 29 November 2020).

8. African Union, Africa CDC. COVID-19 vaccine development and access strategy. $\mathrm{AU}, 2020$. https://africacdc.org/download/covid-19-vaccine-development-andaccess-strategy/ (accessed 30 November 2020).

9. Balet A. What we know about vaccine hesitancy in South Africa. Spotlight, 2020. https://www.spotlightnsp.co.za/2020/10/07/what-we-know-about-vaccinehesitancy-in-south-africa/ (accessed 29 November 2020).

10. Chou WS, Budenz A. Considering emotion in COVID-19 vaccine communication: Addressing vaccine hesitancy and fostering vaccine confidence. https://www. tandfonline.com/doi/full/10.1080/10410236.2020.1838096 (accessed 29 November 2020).

11. Cyranoski D. Why emergency COVID vaccine approvals pose a dilemma for scientists. Nature, 2020. https://www.nature.com/articles/d41586-02003219-y? utm source $=$ Nature + Briefing \&utm campaign $=$ bbb69b275ebriefing-dy-20201123\&utm_medium=email\&utm_term=0_c9dfd39373bbb69b275e-44142809 (accessed 29 November 2020).

12. Nkengasong $J$, Ndembi N Tshangela A, Raji T. COVID-19 vaccines. How to ensure Africa has access. Nature, 2020. https://www.nature.com/articles/d41586-02002774-8 (accessed 29 November 2020). 